\title{
Combining TEM, AFM, and Profilometry for Quantitative Topography Characterization Across All Scales
}

\author{
Abhijeet Gujrati, ${ }^{1 \dagger}$ Subarna R. Khanal, ${ }^{1 \dagger}$ Lars Pastewka, ${ }^{2}$ Tevis D. B. Jacobs ${ }^{1 *}$ \\ 1. Mechanical Engineering and Materials Science, University of Pittsburgh, Pittsburgh, PA \\ 2. Microsystems Engineering, University of Freiburg, Freiburg, Germany \\ $\dagger$ These authors contributed equally to this work * Corresponding author
}

\section{Keywords}

Surface Topography; Power Spectral Density (PSD); Transmission Electron Microscopy (TEM); Atomic Force Microscopy (AFM); Ultrananocrystalline Diamond (UNCD); Surface Roughness Parameters; Self-affinity; Hurst exponent

The published version of this article can be found at: doi: 10.1021/acsami.8b09899 Full citation information is as follows:

A. Gujrati, S.R. Khanal, L. Pastewka, and T.D.B. Jacobs. Combining TEM, AFM, and profilometry for quantitative topography characterization across all scales. ACS Applied Materials \& Interfaces, 10, 29169 (2018)

\begin{abstract}
Surface roughness affects the functional properties of surfaces, including adhesion, friction, hydrophobicity, biological response, and electrical and thermal transport properties. However, experimental investigations to quantify these links are often inconclusive, because surfaces are fractal-like and the values of measured roughness parameters depend on measurement size. Here we demonstrate the characterization of topography of an ultrananocrystalline diamond (UNCD) surface at the Ångström-scale using transmission electron microscopy (TEM), as well as its combination with conventional techniques to achieve a comprehensive surface description spanning eight orders of magnitude in size. We performed more than 100 individual measurements of the nanodiamond film using both TEM and conventional techniques (stylus profilometry and atomic force microscopy). While individual measurements of root-mean-square (RMS) height, RMS slope, and RMS curvature vary by orders of magnitude, we combine the various techniques using the power spectral density (PSD) and use this to compute scale-independent parameters. This analysis reveals that "smooth" UNCD surfaces have an RMS slope greater than one, even larger than the slope of the Austrian Alps when measured on the scale of a human step. This approach of comprehensive multi-scale roughness characterization, measured with Ångström-scale detail, will enable the systematic evaluation and optimization of other technologically relevant surfaces, as well as systematic testing of the many analytical and numerical models for the behavior of rough surfaces.
\end{abstract}




\section{INTRODUCTION}

It was realized more than a century ago ${ }^{1}$ that nominally planar surfaces will only make contact at the points of highest protrusion because of surface roughness. The area of close mechanical contact can thus be thousands of times smaller than the projected, apparent area of the surface. Surface topography therefore strongly affects adhesion, ${ }^{2-4}$ friction, ${ }^{5-7}$ hydrophobicity, ${ }^{8,9}$ biological response, ${ }^{10}$ and electrical ${ }^{11}$ and thermal ${ }^{12}$ transport properties. Surface roughness exists even on polished and patterned surfaces, and may be unavoidable for polycrystalline materials. ${ }^{13}$ Therefore, surface topography and surface finish are widely measured and reported, with international standards specifying different levels of allowable height variation for specific applications (e.g., ASME B46, ${ }^{14}$ ISO 4287, ${ }^{15}$ and ISO $4288{ }^{16}$ ). However, significant questions remain about the character of roughness and how to quantitatively describe its effect on surface functional properties.

Many experimental investigations attempt to correlate surface performance to a single statistical parameter of roughness. For example, a 2013 review about bone marrow stromal cells on ceramic materials concluded that "overall, the effect of surface roughness (at both the nano- and micronscales) and grain size on cell adhesion is inconclusive due to contradicting data in the literature". ${ }^{17}$ The review cites investigations showing adhesion increasing ${ }^{18,19}$ and decreasing ${ }^{20}$ with average roughness, as well as other data showing no trend. ${ }^{21}$ Fifty years ago, Mandelbrot ${ }^{22}$ provided an explanation. His paper, titled "How Long is the Coast of Britain?", showed that there is no singlevalued answer to the title's question. The measured length $L_{\text {coast }}$ of a coastline depends on the length $l$ of the yardstick used to measure it. Rough surfaces exhibit similar scaling laws and analogously, the measured scalar parameters such as root-mean-square (RMS) height are strongly dependent on measurement resolution and scan size. ${ }^{23}$

Analytical $^{2,24-27}$ and numerical ${ }^{28-30}$ models account for the self-affine nature of surfaces and suggest that surface properties can be predicted from a complete statistical description of the surface in the form of the power spectral density (PSD) ${ }^{31}$ or the autocorrelation function (ACF) ${ }^{32}$. These same investigations suggest that adhesion, friction, and contact stiffness depend on the true values of RMS height $h_{r m s}$, RMS slope $h_{r m s}$, and in the case of adhesion ${ }^{2}$ also RMS curvature $h{ }^{\prime}{ }_{r m s}$. This leads to a central challenge in roughness analysis: functional properties of a surface depend on the true scale-spanning topography, while any experimental measurement of topography is inherently technique-dependent and incomplete. The resolution to this challenge is to combine measurements across length scales and, unlike with Mandelbrot's coastlines, to measure the smallest sizes where scaling laws must break down.

\section{RESULTS}

Here, transmission electron microscopy is used to achieve these smallest-size-scale measurements for ultrananocrystalline diamond. Specifically, a 2- $\mu \mathrm{m}$ film was deposited (see Methods) onto a thin-wedge TEM substrate and 79 side-view images of the surface were taken on scales from 
hundreds of nanometers down to Ångströms (Fig. 1a-c). Each image was post-processed to digitize the contour of the surface, thereby extracting line profiles. These profiles were then analyzed as topographic line contours, analogous to measurements from a stylus profilometer or a line-scan from an AFM. Values of commonly used roughness parameters were computed, including RMS height $h_{\text {rms }}$, RMS slope $h^{\prime}$ rms, and RMS curvature $h{ }^{\prime}{ }_{\text {rms }}$, as well as the full surface area $A_{\text {surf }}$. These TEM-based measurements were also used to compute the power spectral density of topography for a statistical description of the surface. The $\mathrm{PSD}^{31,33}$ is the Fourier transform of the height autocorrelation function and mathematically decomposes the overall topography into contributions from different length scales $\lambda$ (typically expressed as a function of wavevector $q=2 \pi / \lambda$ ). See Methods for details of the TEM measurement and image analysis, as well as the calculation of roughness parameters and PSDs. Most importantly, the TEM-measured PSD of the UNCD film (Fig. 1d) enables characterization of topography over lateral length scales ranging from tens-ofnanometers down to $4 \AA$-a regime of roughness that has not been measured in prior investigations of UNCD (e.g., Ref. ${ }^{34}$ ) and which is not accessible using any conventional technique for topography measurement. ${ }^{31}$
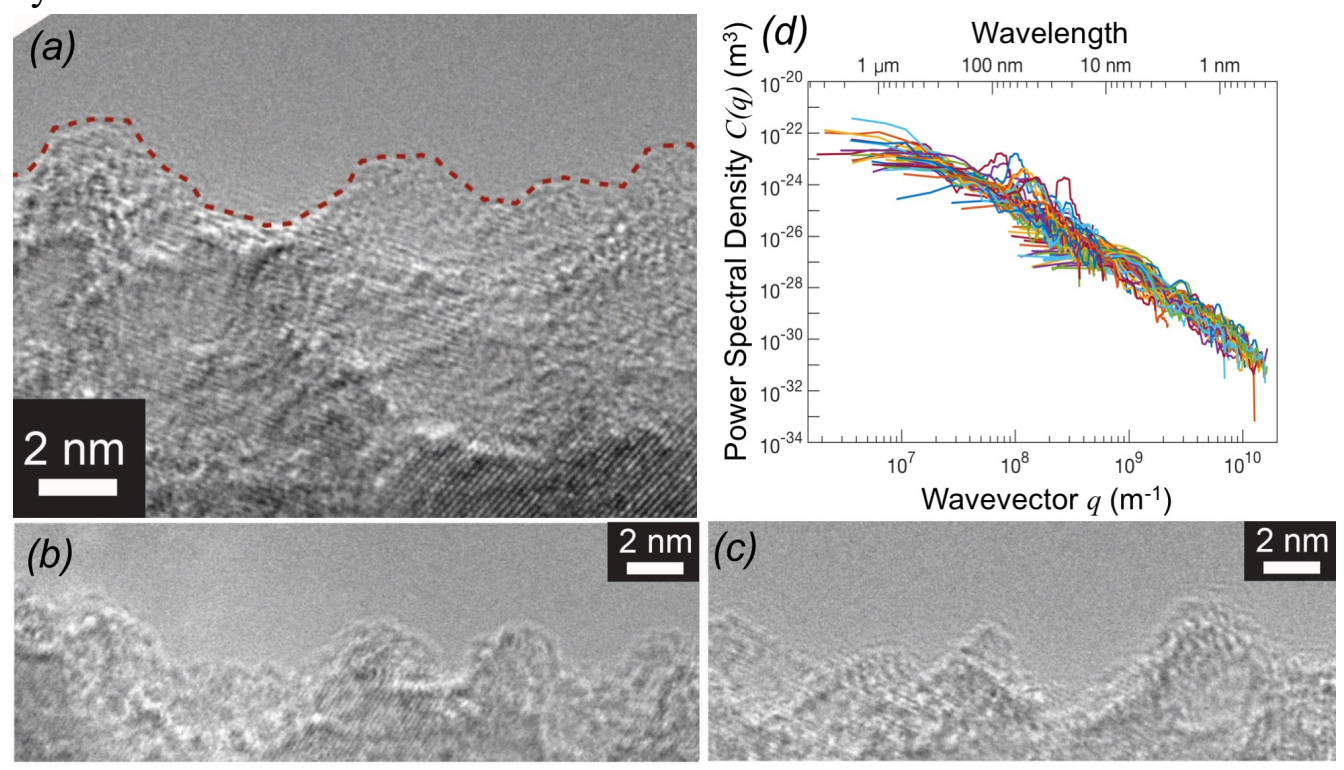

Figure 1: Transmission electron microscopy reveals the surface structure at the Ångström-scale. The topography was captured using side-view TEM images with a wide range of magnifications, resulting in image sizes ranging from over a micron to less than $10 \mathrm{~nm}$. Representative high-resolution images are shown (a-c). The topography was extracted from the outermost contour of the surface (red dashed line in panel a). The power spectral density (d) was computed from each measurement of topography at various magnifications. Overall, the TEM measurements yield topography in the size range between tens-ofnanometers and a few Ångströms, which is inaccessible using conventional topography measurement techniques.

Next the UNCD film was measured using conventional surface topography techniques, for the purposes of combining with the TEM measurements, in order to create a comprehensive description of the surface. The UNCD film was characterized (see Methods) using stylus 
profilometry and atomic force microscopy (AFM), as shown in Fig. 2a-c. The measurements included 20 stylus scans with sizes from 0.3 to $10 \mathrm{~mm}$, and 28 AFM scans with sizes from $100 \mathrm{~nm}$ to $50 \mu \mathrm{m}$. Further, we performed a quantitative analysis of tip-size artifacts following the approach of Refs. 31,35 (as described in Methods). This analysis demonstrates a strong tip effect, especially at the smaller length scales. For stylus measurements (Fig. 2d), the entire region that appears to show self-affine scaling was artifacted and thus unreliable. The AFM data also showed significant sensitivity to tip-radius effects, which were exacerbated by wear-induced tip blunting. Even using best-practices (tapping mode with low tip wear, and accounting for the reliability cut-off), conventional surface characterization techniques were unable to capture the roughness of UNCD at lateral length scales smaller than tens of nanometers. While AFM (and also scanning tunneling microscopy) techniques can achieve Ångström-scale resolution on atomically-flat samples, ${ }^{36}$ for most engineering surfaces the interaction of the tip size and the surface roughness imposes a minimum lateral length scale for reliable measurement. These tip-size artifacts in topography measurements underscore the need for combination with TEM-based Ångström-scale measurements to accurately characterize a surface across all scales.
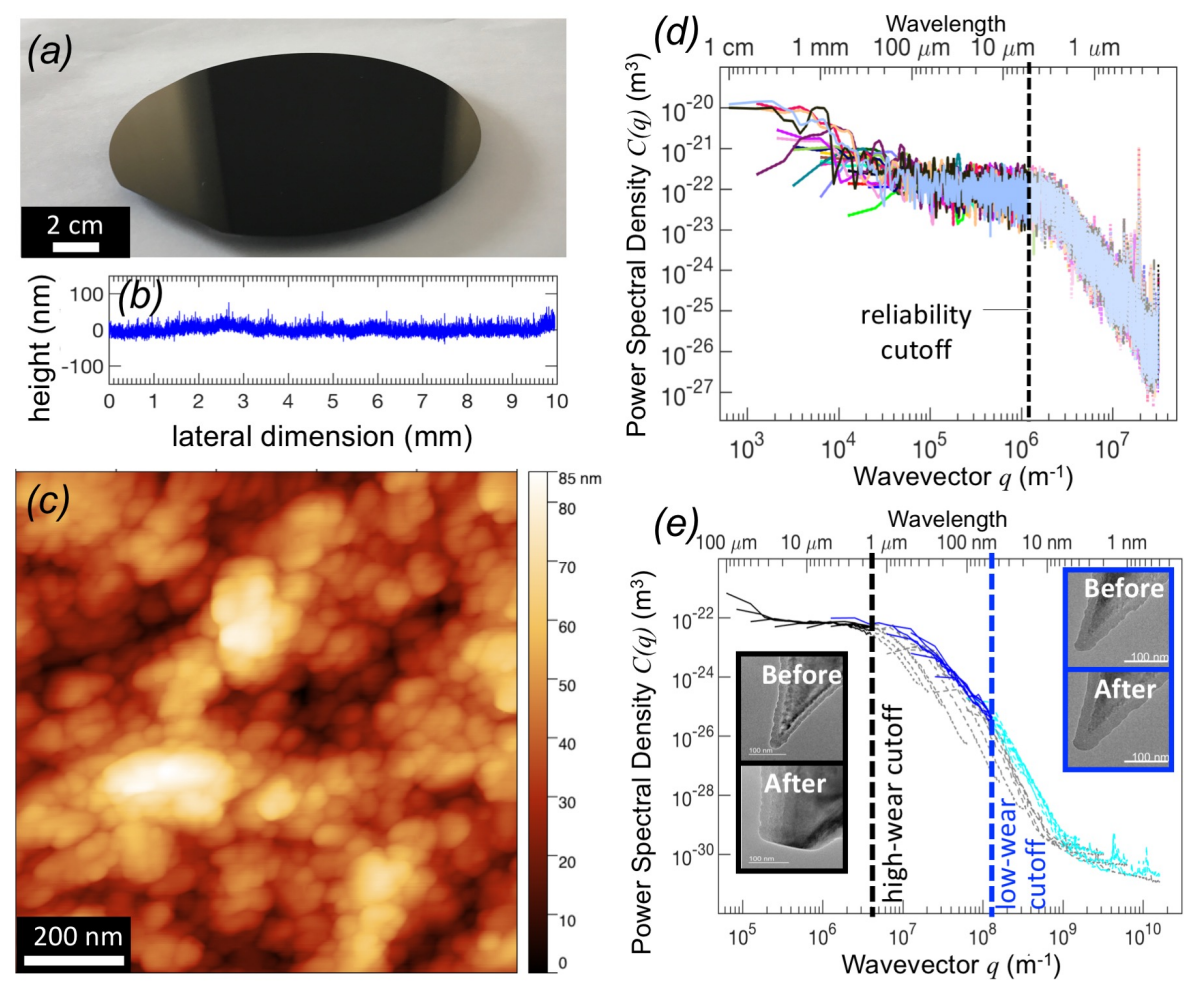

Figure 2: Complementary topography characterization is performed using conventional techniques, and underscores the unreliability of measurements at the smallest lateral length scales. The UNCD film, which has a mirror-like external appearance (a), was characterized using stylus profilometry (b) and AFM (c). The power spectral density (d, e) was computed and tip-size artifacts accounted for (see Methods). For stylus data, the PSD of each measurement is shown; for AFM data, each curve represents an average of all linescans taken in a single square measurement. The left-most portions of the measured PSD curves (solid, darker-color lines) are considered reliable; beyond the reliability cutoff (dashed vertical line), the remaining 
data (dashed, lighter-color lines) is considered artifacted. For the stylus measurements, the entire region that appears to be self-affine is unreliable. The AFM-based PSDs enable measurements to smaller sizes, but tip wear can reduce reliable lateral resolution by at least an order of magnitude. Tip wear was detected using TEM images of the tip taken before and after use (insets in (e)). Even the lowest-wear probes could not accurately characterize the statistics of topography for UNCD at lateral length scales below several tens of nanometers.

\section{DISCUSSION}

The first key outcome from this investigation is the measurement of commonly-used roughness parameters at the smallest length scales. The calculation of roughness parameters is described in the Methods section. Across all techniques, the individual measurements of RMS height, RMS slope, and RMS curvature vary by orders of magnitude with size scale, as shown in Fig. 3a-c. For example, the magnitude of the RMS slope - a critical parameter in numerical and analytical models $^{28-30}$ of rough-surface properties - varies by more than two orders of magnitude as a function of yardstick size (pixel size) $l$. At the largest scale, the stylus profilometer measured an average RMS slope of 0.03 , corresponding to an angle of just $1.7^{\circ}$, reflecting the fact that the UNCD has the appearance equivalent to a polished silicon wafer (see Fig. 1a). At the smallest scales that are accessible by conventional techniques, the AFM data showed an average RMS slope of 0.27 ( $15^{\circ}$ from horizontal), in rough agreement with prior measurements on related films. ${ }^{37}$ However, the novel TEM measurements showed an average value of RMS slope equal to 0.92 ( $43^{\circ}$ from horizontal), more than triple the AFM-measured value. For comparison, the mean surface slope of the UNCD, when measured with high resolution, exceeds the average RMS slope of the Austrian Alps (0.8) when measured with a yardstick on the order of a human step length. ${ }^{38}$ The RMS curvature (Fig. 3c) showed yet a greater influence of measurement parameters, with five orders of magnitude in difference between the smallest and largest values measured. Likewise, the true surface area (the most analogous parameter to Mandelbrot's coastline length) showed significant variation (Fig. 3d). While the stylus data suggested a roughness-induced increase in surface area of just $0.1 \%$ above the projected area, the TEM data demonstrated that the true surface area is approximately double the projected value. Taken together, the variation in these measurements conclusively demonstrate the impossibility of linking any single scalar measurement of topography to a functional property, and suggests a primary cause of the inconclusive results that are widely reported in roughness literature (for example, Ref. 17). 

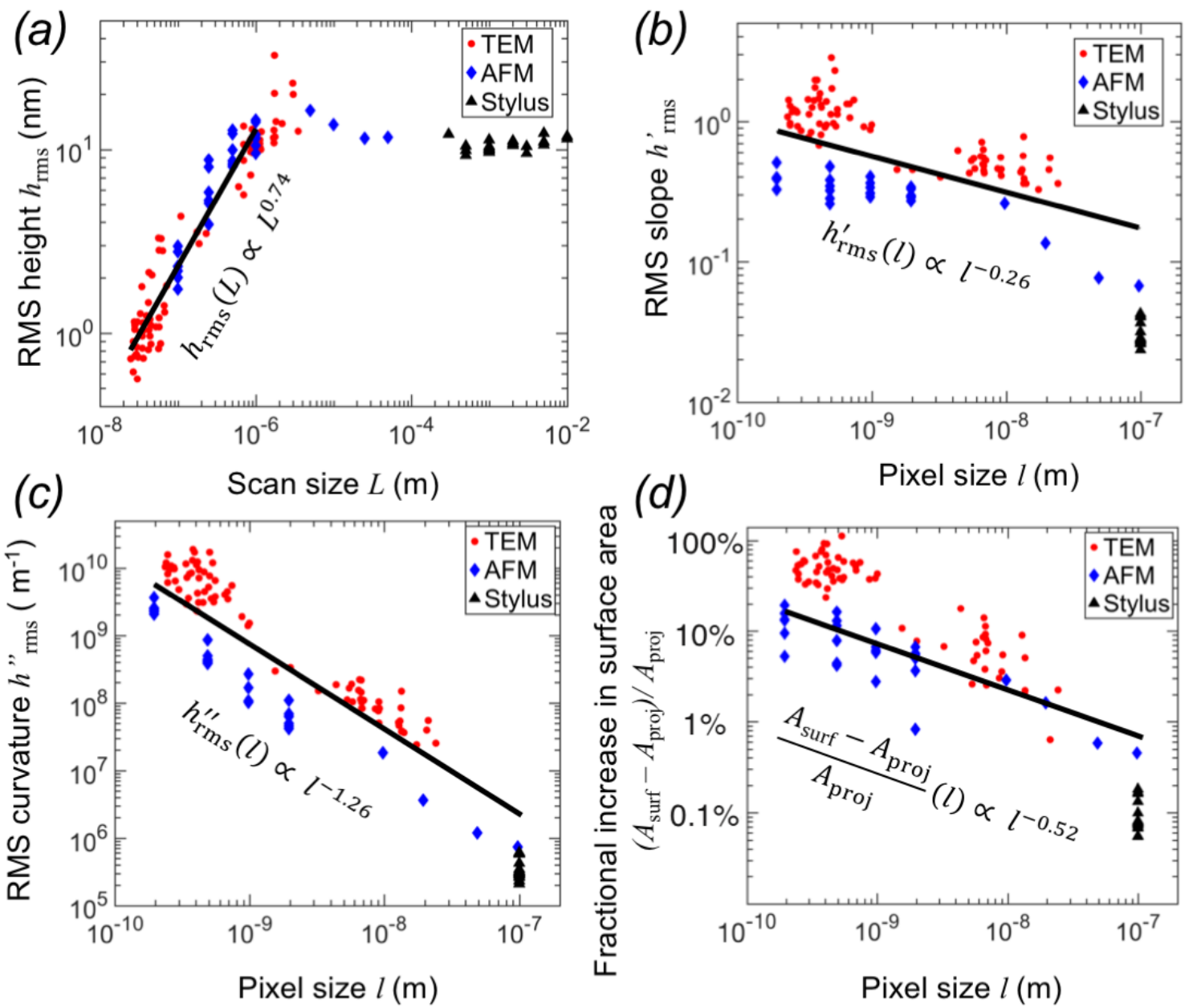

Figure 3: Measurements of scalar roughness parameters of the UNCD show variation by orders of magnitude both within and between experimental techniques. The RMS height (a) varies by more than an order of magnitude depending on how it is measured. Here, the RMS height is presented as a function of scan size, because the largest features have the most significant effect. The solid line shows the best-fit power-law exponent, which corresponds to the Hurst exponent in the variable-bandwidth model (see main text); this value is used to predict the scaling behavior of the other roughness parameters (b-d, solid lines). The RMS slope (b), RMS curvature (c), and computed fractional increase in surface area (d) all show systematic variation by at least two orders of magnitude with measurement size. These parameters are presented as a function of pixel size because the smallest-size features have the most significant effect.

More quantitatively, the scalar roughness parameters can be analyzed as a function of measurement size. Results are analyzed in the style of the variable-bandwidth method (VBM). ${ }^{39,40}$ For a self-affine surface, measured values of $h_{\text {rms }}$ should scale with scan size $L$ according to $h_{\text {rms }}(L) \propto L^{H}$, where $H$ is the Hurst exponent. ${ }^{39}$ Since measurements were taken at a wide range of scan sizes, this sets the upper limit of wavevector for each calculation. For this analysis, we therefore do not need to numerically restrict the bandwidth of our measurements, ${ }^{39}$ but rather rely on their natural bandwidths. The individual measurements of RMS height $h_{\mathrm{rms}}(L)$ (Fig 3a) 
demonstrate power-law scaling corresponding with a Hurst exponent of $H=0.74 \pm 0.05$ at small $L$ and a crossover to a plateau at approximately $L=1 \mu \mathrm{m}$. The RMS slope and RMS curvature of a self-affine surface are predicted ${ }^{27,31}$ to scale as: $h_{\mathrm{rms}}^{\prime}(l) \propto l^{H-1}$ and $h_{\mathrm{rms}}^{\prime \prime}(l) \propto l^{H-2}$, but these trends had not previously been demonstrated experimentally. The present results demonstrate this scaling behavior of these parameters and show that it is in reasonable agreement with the expected behavior for $H=0.74$ (extracted from Fig. 3a). The plateau at $1 \mu \mathrm{m}$ does not appear in Figs. 3b-d because the pixel size of all measurements is below this scale. We show (Methods) that the fractional increase in surface area for a self-affine surface should scale as $\left(A_{\text {surf }}-A_{\text {proj }}\right) / A_{\text {proj }} \propto$ $l^{2 H-2}$; Fig. $3 \mathrm{~d}$ presents the data in this form. Although it has been shown that roughness parameters vary with scan size, this investigation demonstrates the functional form of the scaling for RMS slope, RMS curvature, and true surface area. Further, the TEM measurements enable the calculation of parameters at the very smallest scales.

The second key outcome from this investigation is the calculation of true, scale-invariant roughness parameters. As mentioned, analytical and numerical models ${ }^{2,24-30}$ suggest that adhesion, friction, and contact stiffness depend on the true RMS height, RMS slope, and RMS curvature of the surface. However, Fig. 3 demonstrated the variability of any individual measurement of these parameters. To overcome this paradox, we combine all of the present measurements, spanning eight orders of magnitude in size scale, together into one complete description of the surface. The power spectral density is used as the tool for combining these multi-scale measurements into a single description. Figure 4 shows the computed PSDs from all measurements, with unreliable data not included. The 127 individual measurements, taken at more than 30 different magnifications using three different instruments, collapse onto a single curve. Only three measurements, taken with the same AFM probe, showed deviation (cyan data in Fig. 4) and these are attributed to tip-size artifacts (see Methods). The arithmetic average of all measurements comprises a single function that fully describes the topography for the UNCD surface. 


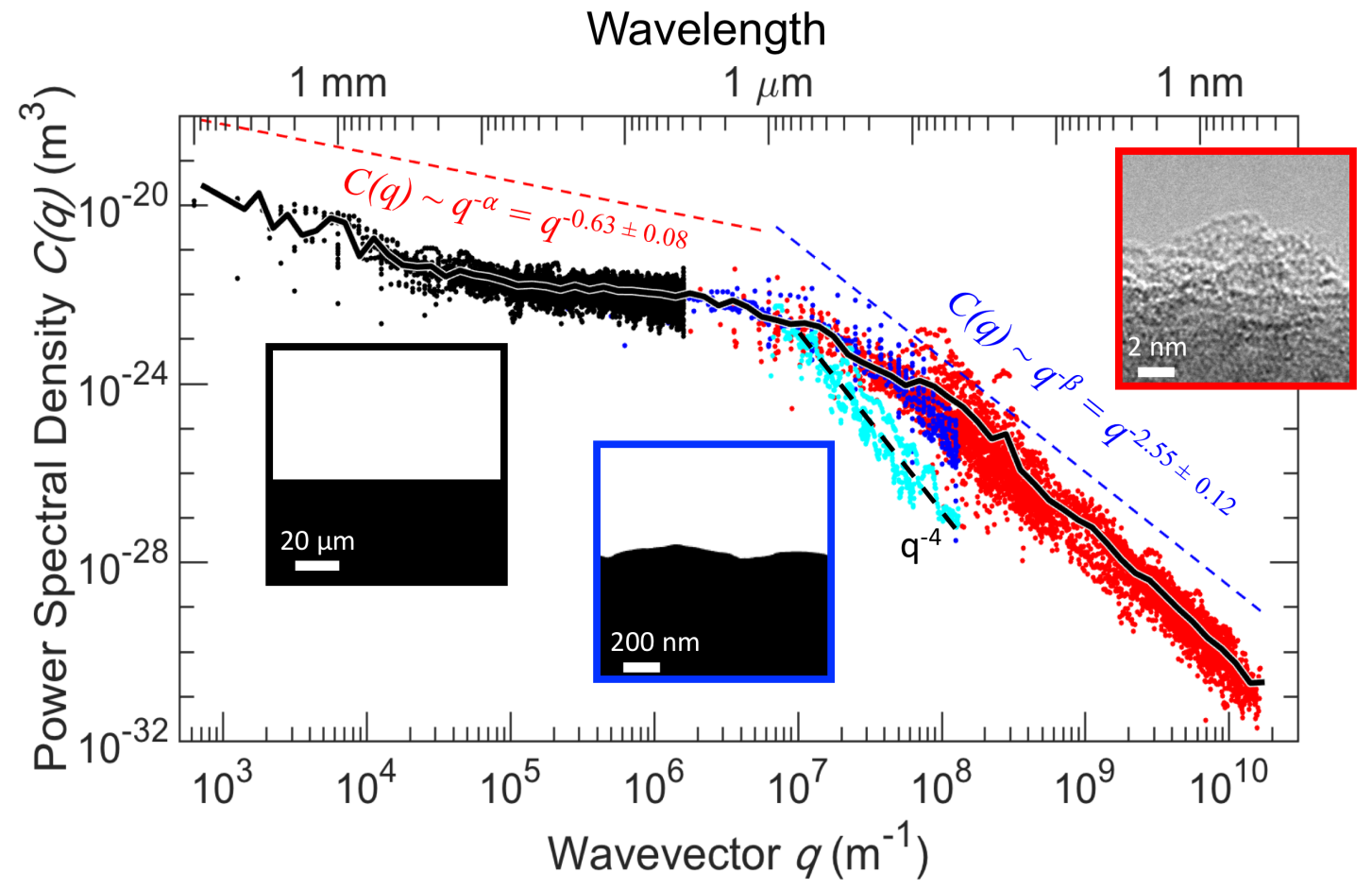

Figure 4: Topography measurements across eight orders of magnitude in length scale are combined into a single description of the surface. The power spectral densities of all 127 measurements at various resolutions were combined into a single plot. The reliable portions of line-scan measurements from stylus (black), AFM (blue), and TEM (red) are shown as points. The cyan data is affected by tip-size artifacts (see Methods). Representative side-view images are shown at 1-to-1 scaling in the insets, which are outlined in the corresponding color. The individual measurements are combined into a single PSD (black line with white outline), which represents a comprehensive description of surface topography. The curve can be approximately fit using power-law scaling relations (colored dashed lines). However, having an experimental measurement of the full multi-resolution PSD curve eliminates the need for assumptions about self-affinity.

The curve shows two regions of approximately power-law scaling with exponent $\alpha=0.63$ at small $q$ (long wavelengths) and $\beta=2.55$ at large $q$ (short wavelengths). The exponents $\alpha$ and $\beta$ extracted from the multi-resolution PSD therefore characterize the long-wavelength and shortwavelength behavior of the UNCD surface, respectively. The cutoff between large and small $q$ is somewhat arbitrary; here it has been chosen as $1 \mu \mathrm{m}$, in accordance with Fig. 3a. It is common in surface topography analysis to consider the large-q portion as the "self-affine" region, and to consider the small-q portion as the "roll-off" region, ${ }^{41}$ where the self-affinity breaks down. Here we will discuss the two regions separately.

In the large-q "self-affine" region, the fractal dimension can be extracted as $D=$ $(2 n+3-\beta) / 2,{ }^{42,43}$ where $n$ is the dimension of space. In the present case of line-scan topography measurements, $n=1$ so $D=(5-\beta) / 2$. For self-affine processes, the fractal 
dimension $D$ is related to the Hurst exponent ${ }^{43,44}$ by $D+H=n+1=2$, therefore a value of Hurst exponent can be extracted from the large-q region using $H=2-D=(\beta-1) / 2=0.77 \pm 0.06$. This value agrees well with the value of $0.74 \pm 0.05$ extracted using the VBM. (Though numerical simulations ${ }^{39,45}$ have shown that fitting Hurst exponents to power-laws can lead to a systematic error of up to 0.2 with the method of calculation.) The measured values of $H$ are consistent with many experimental observations on scales from mountain ranges ${ }^{46}$ to atoms ${ }^{47}$ and processes such as fracture ${ }^{48-50}$ and plastic deformation ${ }^{51}$. A value of $H>0.5$ indicates persistent behavior of the spatial correlations, i.e., a high value is likely to be followed by another high value. We note that our UNCD films are grown on a surface and that common growth models (e.g., EdwardsWilkinson ${ }^{52}$ or Kardar-Parisi-Zhang ${ }^{53}$ ) predict $H=0.5$, but $H>0.5$ is commonly observed in deposition from vapor ${ }^{54}$ or liquid ${ }^{55}$.

We now consider the analysis of the "roll-off" region, but unlike a typical ${ }^{41}$ assumption of constant power below some critical wavevector, we use the framework of fractional Gaussian noise (FGN) ${ }^{43,56,57}$ In this, the fractal dimension $D_{F G N}$ is extracted from the large-q region using the same equation described above; however, the Hurst exponent $H_{F G N}$ is computed from the small-q region as $H_{F G N}=(\alpha+1) / 2 .{ }^{43}$ This analysis yields a value $H_{F G N}=0.82 \pm 0.04$, which agrees with the large- $q$ (self-affine) value of $H$ within experimental uncertainty. The typically-used assumption of constant power in the roll-off region implies a white-noise process; mathematically, this gives $H_{F G N}=0.5$. This assumption is not borne out by the measurements of UNCD, which instead show an FGN Hurst exponent that is approximately equal to the one computed from the large-q region. At present, it is unclear to us why fractional Gaussian noise seems to serve as a good model for the power spectral densities of surface topography. However, the results may indicate that the topography of the long-wavelength "roll-off" region is related to the topography of the short-wavelength "self-affine" region. Specifically, the former has the statistical structure of the derivative of the latter, such that $\alpha=\beta-2$. Additional work is required to verify the generalizability of these relationships between the large-q and small-q regions for surface topography.

Finally, we note that the PSD curve is not completely linear in either the large- or small- $q$ region, and therefore the exact value of the measured scaling exponents will depend on the size scale over which they are measured. For example, if a line is fit to only the largest-q portion of the curve $\left(q>10^{8}\right)$, then the values shift to $D=1.0 \pm 0.04$ and $H=1.0 \pm 0.04$. In general, to eliminate uncertainty in how the curve is fit and to eliminate the need for models of random processes, the following paragraph describes the use of the entire PSD curve (without assumptions of selfaffinity) to compute scale-independent roughness parameters.

The combined data from all measurements (Fig. 4) were used to compute scale-independent values of $h_{r m s}, h^{\prime}{ }_{r m s}$, and $h^{\prime \prime}{ }_{r m s}$, which describe the underlying surface. These roughness parameters are 
equal to the zeroth, second, and fourth moments of the PSD, respectively. ${ }^{31}$ For a one-dimensional PSD, these parameters are computed as follows:

$$
\left(h_{\mathrm{rms}}\right)^{2}=\frac{1}{\pi} \int_{0}^{\infty} C(q) d q, \quad\left(h_{\mathrm{rms}}^{\prime}\right)^{2}=\frac{1}{\pi} \int_{0}^{\infty} q^{2} C(q) d q, \quad\left(h_{\mathrm{rms}}^{\prime \prime}\right)^{2}=\frac{1}{\pi} \int_{0}^{\infty} q^{4} C(q) d q
$$

These integrations were performed numerically using the trapezoidal method, eliminating the need for assumptions about self-affinity. The bounds of integration are defined by the largest measured scan size $(1 \mathrm{~cm})$ and the smallest pixel size (4 $\AA$ ). No smaller-size contribution is expected because this is approaching the atomic scale, beyond which topography is not clearly defined. Using this approach, the computed true values of roughness parameters for the surface were $h_{r m s}=$ $17.5 \pm 1.3 \mathrm{~nm}, h^{\prime}{ }_{r m s}=1.2 \pm 0.28$ equivalent to an angle of $50^{\circ}$, and $h^{\prime}{ }^{\prime}{ }_{r m s}=6.3 \times 10^{9} \pm 1.2 \times 10^{9} \mathrm{~m}^{-1}$. The uncertainty was computed using standard error propagation as applied to the numerical implementation of Eq. 1. For the $h^{\prime}{ }_{r m s}$ and $h^{\prime}{ }_{r m s}$, which depend on the finest scales of roughness, these results demonstrate that the novel TEM approach yields the most accurate estimates of RMS slope and curvature, whereas conventional topography techniques err by an order of magnitude or more. The combination of techniques across all scales yields even more robust measurements of roughness parameters than can be achieved by any method in isolation, and this combined approach is necessary to achieve a comprehensive and predictive description of a surface.

On a final note, while measuring the full multi-scale topography is best, this is not always practical. Therefore, we evaluated the estimation of values of RMS slope and RMS curvature that can be computed using only AFM measurements. The AFM-only calculation can be done in two ways, as shown in the Supporting Information (Sect. S-3). The first method, a simple application of Eq. 1 to AFM data, did not yield accurate values. Specifically, when the integration was performed over the average PSD that was computed from all AFM-based measurements (i.e., without accounting for tip-size artifacts nor the reliability cut-off), the RMS slope and RMS curvature were computed to be $1.9 \pm 1.1$ and $2.9 \times 10^{10} \pm 2.25 \times 10^{10} \mathrm{~m}^{-1}$, respectively. Tip artifacts and noise artifacts caused significant deviations in the measured PSDs. However, a second and more sophisticated application of Eq. 1 to AFM data did yield reasonably accurate values of RMS slope and curvature. Specifically, the following process was used: (1) the reliability cut-off was applied to each AFM measurement to eliminate artifacts; (2) the remaining (reliable) data was averaged to create a single PSD; and (3) the average PSD was extrapolated to the maximum wavevector measured with TEM $\left(q=1.6 \times 10^{10}\right)$. The application of Eq. 1 to the resulting extrapolated PSD yields values of RMS slope and RMS curvature of $0.9 \pm 0.1$ and $6.65 \times 10^{9} \pm 1.7 \times 10^{9} \mathrm{~m}^{-1}$, respectively. This RMS slope value is smaller than for the raw AFM data because the extrapolated PSD has a smaller magnitude at high-frequencies than the noisy raw data from the AFM (see Fig. S-3). Overall, the RMS parameters computed from the extrapolated AFM data are closer to the values computed in the previous paragraph. However, the robustness and generalizability of this extrapolation approach is unknown. Therefore, where possible, we believe that the best way to characterize a surface is to combine many techniques over many length-scales and to include characterization down to the Ångström scale, thus eliminating the need for self-affine assumptions or approximations. 
In conclusion, by characterizing a surface across eight orders of magnitude in size scale, this investigation demonstrates two key advancements in our understanding of surface topography. First, while individual measurements of such critical roughness parameters as RMS slope and RMS curvature will vary by orders of magnitude between and within different experimental techniques, TEM-based measurements are able to approximate the true values for the surface by capturing the smallest-scale topography. For a smooth, conformal UNCD film, the measured value of RMS slope is of order unity and the value of RMS curvature corresponds to an Ångström-scale radius; these values are significantly larger than those that have been measured previously with conventional techniques. Second, we demonstrate the combination of topography measurements across all size scales to compute true, scale-independent parameters for the underlying surface. By using the power spectral density, we show that the more than 127 individual measurements collapse to a single curve. The resulting curve is approximately self-affine at large wavevectors, with a Hurst exponent of 0.77 . We show a separate interpretation of the long-wavelength portion of the PSD (often designated the "roll-off" regime) using fractional Gaussian noise. We further show that, instead of relying on self-affine approximations, one can use the combined multiresolution PSD curve to compute scalar roughness parameters for the surface: RMS height, RMS slope, RMS curvature, and also the roughness-induced increase in true surface area. In summary, although the specific, measured values of the PSD and roughness parameters are unique to this material (unpolished UNCD), the present approach to characterization and analysis is widely generalizable to the topography analysis of many materials. The demonstrated approach enables more accurate evaluation of surface topography of real-world components, and more meaningful correlation with surface properties. Further, it allows the systematic testing of the many existing analytical and numerical models, in order to uncover the physical links between surface topography and surface function.

\section{METHODS}

\section{Material deposition}

Conductive ultrananocrystalline diamond (UNCD) was deposited (by Advanced Diamond Technologies, Romeoville, IL) using a tungsten hot-filament chemical vapor deposition (HFCVD) system with parameters as described in Ref. ${ }^{58}$. An H-rich gas mixture was used, with chamber pressure of 5 Torr and a substrate temperature of $750^{\circ} \mathrm{C}$. The ratio of boron to carbon was maintained at $0.3 \mathrm{at} \%$, to achieve high conductivity in the final film. A $2-\mu \mathrm{m}$-thick film of UNCD was deposited in the same batch on polished silicon wafers and microfabricated silicon wedges. The latter samples are electron transparent and facilitate imaging in the transmission electron microscope. 
Topography characterization using the transmission electron microscope

The small-scale topography was imaged using a TEM (2100F, JEOL, Tokyo, Japan) at an electron accelerating voltage of $200 \mathrm{keV}$. The chosen geometry, a coating of UNCD on a thin microfabricated wedge of silicon, enabled the side-view imaging of the UNCD surface. Eight distinct regions of UCND were imaged, at various magnification levels from 5000x to 800,000x, which corresponds to sampling sizes ranging from $1 \mu \mathrm{m}$ to $25 \mathrm{~nm}$, respectively. The images were taken with a 2000x2000-pixel camera; however, the "pixel size" in the VBM-like analysis corresponded to the average separation between measured points in the traced profiles. While the microscope has 0.22 -nm point-to-point resolution at the highest magnifications, the pixel size of an image scales inversely with magnification.

To extract the surface contour, the TEM images were traced using custom Matlab scripts that create a digitized line profile based on a series of points selected by the user. As shown in Fig. 5a,b, the TEM image is rotated so that the surface is approximately horizontal, then the boundary of the UNCD material is measured. The vast majority of the measured surface was not re-entrant and thus obeyed the rules of a well-defined function, i.e., the height (y-axis) is singlevalued for each horizontal position (x-axis). In rare cases where two adjacent points were captured with identical or decreasing horizontal position, the latter point was removed (Fig. 5c). In just four out of the 79 measurements, there were small portions of the profile that showed backward-bending regions. This backward-bending character is not necessarily physically meaningful as it depends on the rotation of the TEM image during image analysis. Because these regions preclude mathematical analyses that require well-behaved functions, these backwardbending regions were excluded from analysis.
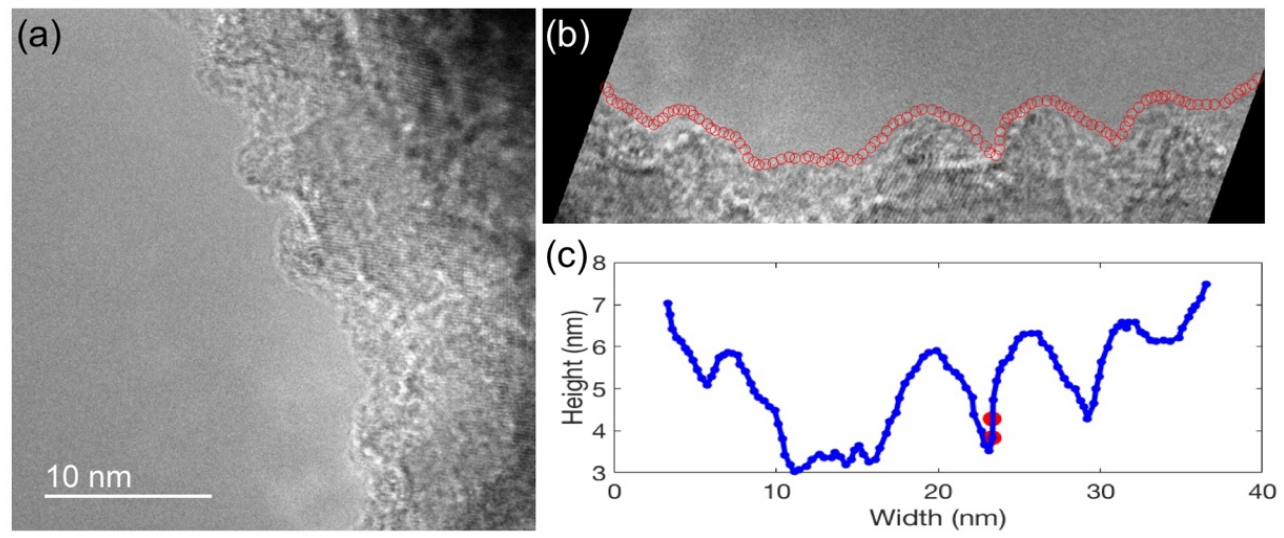

Figure 5: The side-view TEM images of the surface (a) were analyzed using custom algorithms which allowed the tracing of points (red circles) describing the contour of the material (b). These points were digitized and, using the pixel-size calibration of the image, converted to real-space units (c). The rare points that have identical or decreasing x-position from their left-most neighbor (an example is shown in red in (c)) were excluded from the profile. 
Topography characterization using stylus profilometry and atomic force microscopy

The largest scales of topography on the UNCD were measured using one-dimensional line scans with a stylus profilometer (Alpha Step IQ, KLA Tencor, Milpitas, CA) with a 5- $\mu$ m diamond tip. Data were collected at a scanning speed of $10 \mu \mathrm{m} / \mathrm{s}$, with data points every $100 \mathrm{~nm}$. A total of 20 measurements were taken at seven different scan lengths: $0.3,0.5,1,2,3,5$ and $10 \mathrm{~mm}$. These measurements were taken at random orientations of the sample, and did not show meaningful variations with direction. A parabolic correction was applied to all measurements which removed the tilt of the sample and the bowing artifact from the stylus tool. At the largest scan sizes $(3 \mathrm{~mm}$ and larger), a consistent non-parabolic trend was present on all measurements. This was corrected by performing reference scans on polished silicon wafers and subtracting the averaged profiles from all measurements.

The same surface was also measured using an AFM (Dimension V, Bruker, Billerica, MA) in tapping mode with diamond-like carbon-coated probes (Tap DLC300, Mikromasch, Watsonville, CA). A total of 28 measurements were taken at eight different scan sizes: 0.1, 0.25, 0.5, 1, 5, 10, 25 , and $50 \mu \mathrm{m}$. The scanning speed was maintained at $1 \mu \mathrm{m} / \mathrm{s}$ for all scans below $10 \mu \mathrm{m}$, otherwise a scan rate of $0.25 \mathrm{~Hz}$ was used. Each scan had 512 lines, with 512 data points per line, corresponding to pixel sizes in the range of 0.2 to $98 \mathrm{~nm}$. The probes were characterized before and after measurement using the fixture and procedure described in Ref. ${ }^{59}$, and had initial tip radii of $17 \pm 4 \mathrm{~nm}$. The first set of measurements resulted in significant wear of the probes, with afteruse radii greater than $120 \mathrm{~nm}$. This was alleviated using the wear-mitigation strategies described in Ref. ${ }^{60}$. In subsequent testing, the values of free-air amplitude and amplitude ratio were kept in the range of $37-49 \mathrm{~nm}$ and $0.15-0.3$, respectively. With these parameters, the tip radii were confirmed to increase only slightly, to an average of $26 \mathrm{~nm}$ after the completion of scanning. While AFM provides a two-dimensional map of surface topography, the data were analyzed as a series of line scans, both to facilitate direct comparison with other techniques and to avoid apparent anisotropy due to instrument drift. For display purposes, Fig. 2 shows the PSD that results from averaging all 512 line scans for a single AFM measurement. By contrast, Fig. 4 shows the PSD from a single representative line scan from each AFM measurement, in order to be consistent with the line-scan data from stylus and TEM.

\section{Computing the power spectral density}

For a line scan with height $h(x)$ over lateral position $x$, the Fourier transform of the surface

topography is given by $\tilde{h}(q)=\int_{0}^{L} h(x) e^{-i q x} d x$. The $\operatorname{PSD}^{31}$ is the Fourier transform of the autocorrelation of $h(x)$ or, equivalently, the square of the amplitude of $\tilde{h}(q)$; i.e., $C(q)=$ $L^{-1}|\tilde{h}(q)|^{2}$. Therefore, by applying the Fourier transform convention described in Ref. 31 to onedimensional line-scan data, $\tilde{h}(q)$ has units of $\left[\mathrm{m}^{2}\right]$ and $C(q)$ (which is designated $\mathrm{C}^{1 \mathrm{D}}$ in Ref. 31) has units of $\left[\mathrm{m}^{3}\right]$. 
The combined PSD represents the arithmetic average of all of the individual PSDs that were computed from each topography measurement. Because random surfaces are often described as Gaussian random fields (according to the random process model of surface roughness ${ }^{61}$ ) then each value of $\tilde{h}(q)$ should be a complex number that has random real and imaginary components distributed according to a Gaussian distribution. Therefore, $C(q)$ should be distributed according to a $\chi^{2}$-distribution with 2-degrees of freedom, i.e., an exponential distribution. Therefore, the maximum likelihood estimator for true value of $C(q)$ at each value of $\mathrm{q}$ is the arithmetic average of all individual measurements at that value of $q$.

\section{Assessing tip-size artifacts}

The "reliability cutoff" $q_{c}$ above which tip-size artifacts become significant was determined according to the method described in Ref. 31. Specifically, for known radius of curvature $R$ of the imaging tip, the reliability cutoff can be determined for a given scan by finding the value of $q_{c}$ where $h_{\text {rms }}^{\prime \prime}$ is equal to the tip curvature. Following Eq. 1 , the reliability cutoff is computed as follows, where the constant $c$ is set to 0.5 :

$$
\left[h_{r m s}^{\prime \prime}\left(q_{c}\right)\right]^{2}=\frac{1}{\pi} \int_{0}^{q_{c}} q^{4} C(q) d q=\frac{1}{4 R^{2}} .
$$

Most AFM probes were imaged in the TEM after scanning, but one AFM probe broke before this could take place. The last three measurements taken with this probe resulted in the three curves shown in cyan in Fig. 4. While Eq. 2 cannot be applied because the tip radius $R$ is unknown, these

measurements are believed to be artifacted for two reasons. First, they display clear $\mathrm{q}^{-4}$ scaling behavior, which is a hallmark of tip-artifacted one-dimensional PSDs. ${ }^{31}$ Second, these three measurements deviate from the other 56 measurements taken in this region ( 25 from the AFM and 31 from the TEM), as shown in Fig. S2 in the Supporting Information. Therefore, these three measurements are considered to be unreliable where they exhibit $q^{-4}$ scaling and these portions are not included in the calculations.

\section{Computing scalar roughness parameters}

The individual measurements of root-mean-square height, slope, and curvature were computed from each line scan by numerically integrating in real-space:

$$
h_{\mathrm{rms}}^{2}=\frac{1}{L} \int_{0}^{L} h^{2}(x) d x, \quad h_{\mathrm{rms}}^{\prime 2}=\frac{1}{L} \int_{0}^{L}\left(\frac{d h}{d x}\right)^{2} d x, \quad h_{\mathrm{rms}}^{\prime \prime 2}=\frac{1}{L} \int_{0}^{L}\left(\frac{d^{2} h}{d x^{2}}\right)^{2} d x
$$

These general equations for RMS height, slope, and curvature $\left(h_{r m s}, h_{r m s}\right.$, and $h{ }^{\prime}{ }_{r m s}$, respectively) are implemented using the trapezoidal method on a non-uniform grid. While the trapezoidal method is simple and standardized, its implementation here takes on a slightly unique form because of the embedded derivatives. Using a first-order finite-difference approximation for the derivatives, a trapezoidal implementation of Eq. 3 takes the following form:

$$
\begin{gathered}
h_{\mathrm{rms}}^{2}=\frac{1}{L} \sum_{i=2}^{N}\left[\frac{1}{2}\left(h_{i}{ }^{2}+h_{i-1}^{2}\right)\left(x_{i}-x_{i-1}\right)\right] \\
h_{\mathrm{rms}}^{\prime 2}=\frac{1}{L\left(\frac{N-1}{N}\right)} \sum_{i=3}^{N}\left[\frac{1}{2}\left(\left(\frac{h_{i}-h_{i-1}}{x_{i}-x_{i-1}}\right)^{2}+\left(\frac{h_{i-1}-h_{i-2}}{x_{i-1}-x_{i-2}}\right)^{2}\right)\left(x_{i}-x_{i-1}\right)\right]
\end{gathered}
$$




$$
h_{\mathrm{rms}}^{\prime \prime 2}=\frac{1}{L\left(\frac{N-2}{N}\right)} \sum_{i=4}^{N}\left[\frac{1}{2}\left(\left(\frac{\frac{h_{i}-h_{i-1}}{x_{i}-x_{i-1}}-\frac{h_{i-1}-h_{i-2}}{x_{i-1}-x_{i-2}}}{\left(x_{i}-x_{i-2}\right) / 2}\right)^{2}+\left(\frac{\frac{h_{i-1}-h_{i-2}}{x_{i-1}-x_{i-2}}-\frac{h_{i-2}-h_{i-3}}{x_{i-2}-x_{i-3}}}{\left(x_{i-1}-x_{i-3}\right) / 2}\right)^{2}\right)\left(x_{i-1}-x_{i-2}\right)\right]
$$

where $N$ measurements were made over a scan size $L$, and the $i^{\text {th }}$ measurement of height $h_{i}$ was measured at horizontal position $x_{i}$.

We compute the ratio of full surface area $A_{\text {surf }}$ to projected area $A_{\text {proj }}$ from a line scan $h(x)$ of length $L_{x}$ by assuming isotropic surfaces. The equations to do so are derived in the Supporting Information (Sect. S-1), but the final result is as follows:

$$
\begin{aligned}
& \frac{A_{\text {surf }}}{A_{\text {proj }}} \approx \frac{\sqrt{\pi}}{h_{\mathrm{rms}}^{\prime} L_{x}} \int_{0}^{L_{x}}\left|\frac{\partial h}{\partial x}\right| \sqrt{1+\left(\frac{\partial h}{\partial x}\right)^{2}} d x \quad \text { for } h_{\mathrm{rms}}^{\prime}>0.2 \\
& \frac{A_{\text {surf }}}{A_{\text {proj }}} \approx 1+\frac{1}{2} h_{\mathrm{rms}}^{\prime 2} \quad \text { for } h_{\mathrm{rms}}^{\prime}<0.2
\end{aligned}
$$

Equation $5 \mathrm{~b}$ represents the traditional approximation ${ }^{3}$ for the limit of small RMS slope $h_{\mathrm{rms}}^{\prime}$.

\section{ASSOCIATED CONTENT}

\section{Supporting Information}

Section S-1: Derivation of roughness-induced surface area.

Section S-2: A figure that distinguishes the individual PSDs of each measurement (for clarity).

Section S-3: A process for approximating the full, multi-scale RMS parameters using only AFM data.

This Supporting Information is available free of charge on the ACS Publications website at http://pubs.acs.org. (And is appended to the end of this document)

\section{AUTHOR INFORMATION}

*E-mail: tjacobs@pitt.edu

\section{ACKNOWLEDGEMENTS}

The authors thank Nicolaie Moldovan and Hongjun Zeng (Advanced Diamond Technologies) for helpful discussion. Use of the NanoFabrication and Characterization Facility (NFCF) in the Petersen Institute for Nano Science and Engineering (PINSE) is acknowledged. T.D.B.J. acknowledges funding from the National Science Foundation under award CMMI-1727378, and from the University of Pittsburgh, through the Central Research Development Fund (CRDF). L.P. acknowledges funding from the Deutsche Forschungsgemeinschaft under grant PA 2023/2. 


\section{References}

(1) Binder, L. Der Widerstand von Kontakten. Elektrotech. Maschinenbau 1912, 30 (September), 781-782.

(2) Pastewka, L.; Robbins, M. O. Contact between Rough Surfaces and a Criterion for Macroscopic Adhesion. Proc. Natl. Acad. Sci. 2014, 111 (9), 3298-3303.

(3) Persson, B. N. J.; Tosatti, E. The Effect of Surface Roughness on the Adhesion of Elastic Solids. J. Chem. Phys. 2001, 115 (12), 5597.

(4) Fuller, K. N. G.; Tabor, D. The Effect of Surface Roughness on the Adhesion of Elastic Solids. Proc. R. Soc. Lond. A 1975, 345 (1642), 327-342.

(5) Persson, B. N. J.; Sivebaek, I. M.; Samoilov, V. N.; Zhao, K.; Volokitin, A. I.; Zhang, Z. On the Origin of Amonton's Friction Law. J. Phys. Condens. Matter 2008, 20 (39), 395006.

(6) Weber, B.; Suhina, T.; Junge, T.; Pastewka, L.; Brouwer, A. M.; Bonn, D. Molecular Probes Reveal Deviations from Amontons' Law in Multi-Asperity Frictional Contacts. Nat. Commun. 2018, 9, 888.

(7) Carbone, G.; Putignano, C. Rough Viscoelastic Sliding Contact: Theory and Experiments. Phys. Rev. E 2014, 89 (3), 032408.

(8) Davis, A.; Yeong, Y. H.; Steele, A.; Bayer, I. S.; Loth, E. Superhydrophobic Nanocomposite Surface Topography and Ice Adhesion. ACS Appl. Mater. Interfaces 2014, 6 (12), 9272-9279.

(9) Dorrer, C.; Rühe, J. Some Thoughts on Superhydrophobic Wetting. Soft Matter 2009, 5 (1), 51 .

(10) Brunetti, V.; Maiorano, G.; Rizzello, L.; Sorce, B.; Sabella, S.; Cingolani, R.; Pompa, P. P. Neurons Sense Nanoscale Roughness with Nanometer Sensitivity. Proc. Natl. Acad. Sci. 2010, 107 (14), 6264-6269.

(11) Kogut, L.; Komvopoulos, K. Electrical Contact Resistance Theory for Conductive Rough Surfaces. J. Appl. Phys. 2003, 94 (5), 3153-3162.

(12) Gotsmann, B.; Lantz, M. A. Quantized Thermal Transport across Contacts of Rough Surfaces. Nat. Mater. 2013, 12, 59-65.

(13) Zhang, X.; Han, J.; Plombon, J. J.; Sutton, A. P.; Srolovitz, D. J.; Boland, J. J. Nanocrystalline Copper Films Are Never Flat. Science 2017, 357 (6349), 231-254.

(14) ASME. Surface Texture (Surface Roughness, Waviness, and Lay). B46.1 - 2009.

(15) ISO. Geometrical Product Specifications (GPS) -- Surface Texture: Profile Method -Terms, Definitions and Surface Texture Parameters. 4287:1997.

(16) ISO. Geometrical Product Specifications (GPS) -- Surface Texture: Profile Method -Rules and Procedures for the Assessment of Surface Texture. 4288:1996.

(17) Samavedi, S.; Whittington, A. R.; Goldstein, A. S. Calcium Phosphate Ceramics in Bone Tissue Engineering: A Review of Properties and Their Influence on Cell Behavior. Acta Biomater. 2013, 9 (9), 8037-8045. 
(18) Dulgar-Tulloch, A. J.; Bizios, R.; Siegel, R. W. Human Mesenchymal Stem Cell Adhesion and Proliferation in Response to Ceramic Chemistry and Nanoscale Topography. $J$. Biomed. Mater. Res. - Part A 2009, 90 (2), 586-594.

(19) Deligianni, D. D.; Katsala, N. D.; Koutsoukos, P. G.; Missirlis, Y. F. Effect of Surface Roughness of Hydroxyapatite on Human Bone Marrow Cell Adhesion, Proliferation, Differentiation and Detachment Strength. Biomaterials 2000, 22 (1), 87-96.

(20) Zhou, G. S.; Su, Z. Y.; Cai, Y. R.; Liu, Y. K.; Dai, L. C.; Tang, R. K.; Zhang, M. Different Effects of Nanophase and Conventional Hydroxyapatite Thin Films on Attachment, Proliferation and Osteogenic Differentiation of Bone Marrow Derived Mesenchymal Stem Cells. Biomed Mater Eng 2007, 17 (6), 387-395.

(21) Li, X.; van Blitterswijk, C. A.; Feng, Q.; Cui, F.; Watari, F. The Effect of Calcium Phosphate Microstructure on Bone-Related Cells in Vitro. Biomaterials 2008, 29 (23), 3306-3316.

(22) Mandelbrot, B. B. How Long Is the Coast of Britain? Statistical Self-Similarity and Fractional Dimension. Science 1967, 156 (3775), 636-638.

(23) Sayles, R. S.; Thomas, T. R. Surface Topography as a Nonstationary Random Process. Nature 1978, 271 (5644), 431-434.

(24) Persson, B. N. J.; Scaraggi, M. Theory of Adhesion: Role of Surface Roughness. J. Chem. Phys. 2014, 124701 (12), 124701-124715.

(25) Persson, B. N. J. Adhesion between Elastic Bodies with Randomly Rough Surfaces. Phys. Rev. Lett. 2002, 89 (24), 245502.

(26) Bush, A. W.; Gibson, R. D.; Thomas, T. R. The Elastic Contact of a Rough Surface. Wear 1975, 35, 87-111.

(27) Persson, B. N. J.; Albohr, O.; Tartaglino, U.; Volokitin, A. I.; Tosatti, E. On the Nature of Surface Roughness with Application to Contact Mechanics, Sealing, Rubber Friction and Adhesion. J. Phys. Condens. Matter 2005, 17 (1), R1-R62.

(28) Campañá, C.; Müser, M. H. Contact Mechanics of Real vs. Randomly Rough Surfaces: A Green's Function Molecular Dynamics Study. Europhys. Lett. 2007, 77, 38005.

(29) Putignano, C.; Afferrante, L.; Carbone, G.; Demelio, G. The Influence of the Statistical Properties of Self-Affine Surfaces in Elastic Contacts: A Numerical Investigation. $J$. Mech. Phys. Solids 2012, 60 (5), 973-982.

(30) Müser, M. H.; Dapp, W. B.; Bugnicourt, R.; Sainsot, P.; Lesaffre, N.; Lubrecht, T. A.; Persson, B. N. J.; Harris, K.; Bennett, A.; Schulze, K.; et al. Meeting the ContactMechanics Challenge. Tribol. Lett. 2017, 65 (4), 118.

(31) Jacobs, T. D. B.; Junge, T.; Pastewka, L. Quantitative Characterization of Surface Topography Using Spectral Analysis. Surf. Topogr. Metrol. Prop. 2017, 5 (1), 013001.

(32) Wang, A.; Martin, H. M. On the Usefulness of the Height-Difference-Autocorrelation Function for Contact Mechanics. Tribol. Int. 2017, 123, 224-233.

(33) Stover, J. C. Optical Scattering: Measurement and Analysis; SPIE Press, 1995; Vol. 2. 
(34) Podgursky, V.; Bogatov, A.; Sedov, V.; Sildos, I.; Mere, A.; Viljus, M.; Buijnsters, J. G.; Ralchenko, V. Growth Dynamics of Nanocrystalline Diamond Films Produced by Microwave Plasma Enhanced Chemical Vapor Deposition in Methane/Hydrogen/Air Mixture: Scaling Analysis of Surface Morphology. Diam. Relat. Mater. 2015, 58, 172179.

(35) Church, E. L.; Takacs, P. Z. Effects of the Nonvanishing Tip Size in Mechanical Profile Measurements. In Proc. SPIE 1332, Optical Testing and Metrology III: Recent Advances in Industrial Optical Inspection; Grover, C. P., Ed.; International Society for Optics and Photonics, 1991; Vol. 1332, pp 504-514.

(36) Fukuma, T.; Ichii, T.; Kobayashi, K.; Yamada, H.; Matsushige, K. True-Molecular Resolution Imaging by Frequency Modulation Atomic Force Microscopy in Various Environments. Appl. Phys. Lett. 2005, 86 (3), 1-3.

(37) Dychalska, A.; Fabisiak, K.; Paprocki, K.; Makowiecki, J.; Iskaliyeva, A.; Szybowicz, M. A Raman Spectroscopy Study of the Effect of Thermal Treatment on Structural and Photoluminescence Properties of CVD Diamond Films. Mater. Des. 2016, 112, 320-327.

(38) Rott, H. The Analysis of Backscattering Properties from SAR Data of Mountain Regions. IEEE J. Ocean. Eng. 1984, 9 (5), 347-355.

(39) Schmittbuhl, J.; Vilotte, J.-P.; Roux, S. Reliability of Self-Affine Measurements. Phys. Rev. E 1995, 51 (1), 131-147.

(40) Mandelbrot, B. B. Self-Affine Fractals and Fractal Dimension. Phys. Scr. 1985, 32 (4), 257-260.

(41) Persson, B. N. J. Contact Mechanics for Randomly Rough Surfaces. Surf. Sci. Rep. 2006, 61 (4), 201-227.

(42) Orey, S. Gaussian Sample Functions and the Hausdorff Dimension of Level Crossings. Zeitschrift für Wahrscheinlichkeitstheorie und Verwandte Gebiete 1970, 15 (3), 249-256.

(43) Gneiting, T.; Schlather, M. Stochastic Models That Separate Fractal Dimension and the Hurst Effect. SIAM Rev. 2004, 46 (2), 269-282.

(44) Mandelbrot, B. B. The Fractal Geometry of Nature; W. H. Freeman, 1982.

(45) Zhang, X.; Xu, Y.; Jackson, R. L. An Analysis of Generated Fractal and Measured Rough Surfaces in Regards to Their Multi-Scale Structure and Fractal Dimension. Tribol. Int. 2017, 105 (June 2016), 94-101.

(46) Candela, T.; Renard, F.; Klinger, Y.; Mair, K.; Schmittbuhl, J.; Brodsky, E. E. Roughness of Fault Surfaces over Nine Decades of Length Scales. J. Geophys. Res. Solid Earth 2012, 117 (8), B08409.

(47) Persson, B. N. J. On the Fractal Dimension of Rough Surfaces. Tribol. Lett. 2014, 54 (1), 99-106.

(48) Mandelbrot, B. B.; Passoja, D. E.; Paullay, A. J. Fractal Character of Fracture Surfaces of Metals. Nature 1984, 308, 721-722.

(49) Ponson, L.; Bonamy, D.; Bouchaud, E. Two-Dimensional Scaling Properties of Experimental Fracture Surfaces. Phys. Rev. Lett. 2006, 96, 035506. 
(50) Bonamy, D.; Ponson, L.; Prades, S.; Bouchaud, E.; Guillot, C. Scaling Exponents for Fracture Surfaces in Homogeneous Glass and Glassy Ceramics. Phys. Rev. Lett. 2006, 97 (13), 135504.

(51) Zaiser, M.; Grasset, F. M.; Koutsos, V.; Aifantis, E. C. Self-Affine Surface Morphology of Plastically Deformed Metals. Phys. Rev. Lett. 2004, 93 (19), 1-4.

(52) Edwards, S. F.; Wilkinson, D. R. The Surface Statistics of a Granular Aggregate. Proc. $R$. Soc. A Math. Phys. Eng. Sci. 1982, 381 (1780), 17-31.

(53) Kardar, M.; Parisi, G.; Zhang, Y.-C. Dynamic Scaling of Growing Interfaces. Phys. Rev. Lett. 1986, 56 (9), 889.

(54) Yanguas-Gil, A.; Cotrino, J.; Walkiewicz-Pietrzykowska, A.; González-Elipe, A. R. Scaling Behavior and Mechanism of Formation of $\mathrm{SiO} 2$ Thin Films Grown by PlasmaEnhanced Chemical Vapor Deposition. Phys. Rev. B 2007, 76 (7), 075314.

(55) Hasan, N. M.; Mallett, J. J.; dos Santos Filho, S. G.; Pasa, A. A.; Schwarzacher, W. Dynamic Scaling of the Surface Roughness of Cu Deposited Using a Chemical Bath. Phys. Rev. B 2003, 67 (8), 81401.

(56) Mandelbrot, B. B.; van Ness, J. W. Fractional Brownian Motions, Fractional Noises and Applications. SIAM Rev. 1968, 10 (4), 422--437.

(57) Beran, J.; Feng, Y.; Ghosh, S.; Kulik, R. Long-Memory Processes: Probabilistic Theories and Statistical Methods; 2013.

(58) Zeng, H.; Konicek, A. R.; Moldovan, N.; Mangolini, F.; Jacobs, T. D. B.; Wylie, I.; Arumugam, P. U.; Siddiqui, S.; Carpick, R. W.; Carlisle, J. A. Boron-Doped Ultrananocrystalline Diamond Synthesized with an H-Rich/Ar-Lean Gas System. Carbon N. Y. 2015, 84 (1), 103-117.

(59) Jacobs, T. D. B.; Wabiszewski, G. E.; Goodman, A. J.; Carpick, R. W. Characterizing Nanoscale Scanning Probes Using Electron Microscopy: A Novel Fixture and a Practical Guide. Rev. Sci. Instrum. 2016, 87 (1), 13703-13711.

(60) Vahdat, V.; Carpick, R. W. Practical Method to Limit Tip-Sample Contact Stress and Prevent Wear in Amplitude Modulation Atomic Force Microscopy. ACS Nano 2013, 7 (11), 9836-9850.

(61) Nayak, P. R. Random Process Model of Rough Surfaces. J. Lubr. Technol. 1971, 93 (3), 398. 
TOC FIGURE

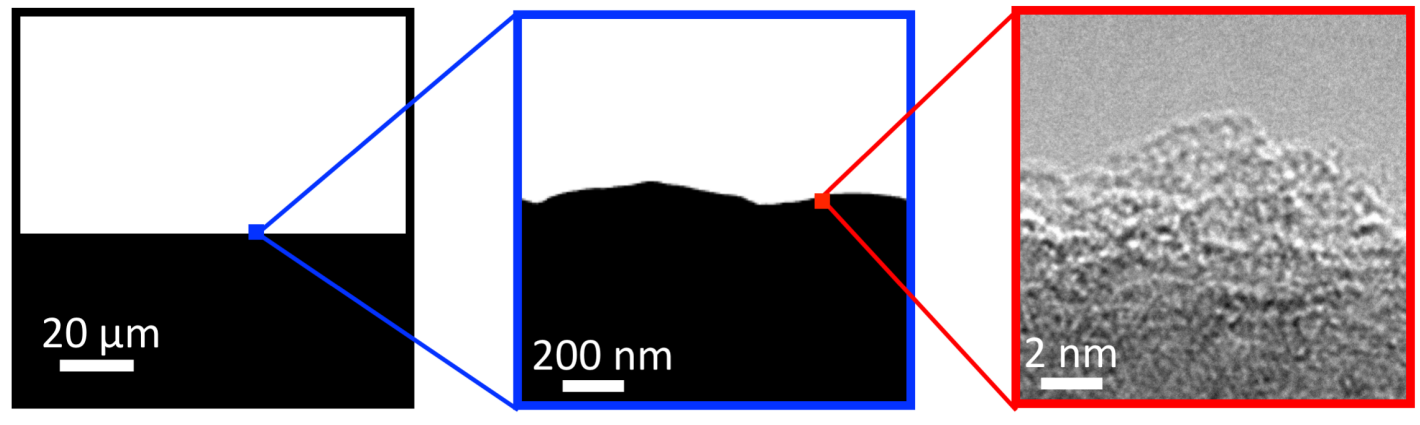


Supporting Information for "Combining TEM, AFM, and Profilometry for Quantitative Topography Characterization Across All Scales"

Authors:

Abhijeet Gujrati, ${ }^{1 \dagger}$ Subarna R. Khanal, ${ }^{1 \dagger}$ Lars Pastewka, ${ }^{2}$ Tevis D. B. Jacobs ${ }^{1 *}$

\section{Affiliations:}

1. Mechanical Engineering and Materials Science, University of Pittsburgh, Pittsburgh, Pennsylvania 15261, United States

2. Microsystems Engineering, University of Freiburg, 79110, Freiburg, Germany

$\dagger$ These authors contributed equally to this work

* Corresponding author

*Email - tjacobs@pitt.edu 


\section{S-1. Derivation of the equation for surface area (Eq. 5 of the main text)}

From a full two-dimensional topography map $h(x, y)$, the surface area $A_{\text {surf }}$ is straightforwardly obtained from the expression:

$$
A_{\text {surf }}=\int \sqrt{1+|\nabla h|^{2}} d x d y=\int \sqrt{1+\left(\frac{\partial h}{\partial x}\right)^{2}+\left(\frac{\partial h}{\partial y}\right)^{2}} d x d y
$$

Equation S1 is the classic result for the arc length, when it is extended to surfaces. For small slopes $\nabla h$ the square root can be expanded into a Taylor series and truncated above quadratic order in $\nabla h$. This gives:

$$
A_{\mathrm{surf}} \approx \int\left(1+\frac{1}{2}|\nabla h|^{2}\right) d x d y=A_{\mathrm{proj}}\left(1+\frac{1}{2} h_{\mathrm{rms}}^{\prime 2}\right)
$$

where $A_{\text {proj }}=\int d x d y=L_{x} L_{y}$, and $L_{x}$ and $L_{y}$ are the linear dimensions of the topography map.

Equation S1 is straightforward to apply for topography maps. Equation S2 can be used to compute the surface areas for line scans $h(x)$ for small slopes. Assuming isotropicity of the surface, we obtain a value for the two-dimensional root-mean-square slope:

$$
h_{\mathrm{rms}}^{\prime 2}=\frac{2}{L_{x}} \int d x\left(\frac{\partial h}{\partial x}\right)^{2}
$$

This approximation breaks down for large slopes. In order to arrive at an expression for large slopes, we first define the slope distribution function:

$$
\phi\left(s_{x}, s_{y}\right)=\frac{1}{A_{\text {proj }}} \int \delta\left(s_{x}-\frac{\partial h(x, y)}{\partial x}\right) \delta\left(s_{y}-\frac{\partial h(x, y)}{\partial y}\right) d x d y
$$

Using $\int f\left(\frac{\partial h}{\partial x}, \frac{\partial h}{\partial y}\right) d x d y=A_{\text {proj }} \int \phi\left(s_{x}, s_{y}\right) f\left(s_{x}, s_{y}\right) d s_{x} d s_{y}$ we can re-express Eq. S1 as: 


$$
\frac{A_{\text {surf }}}{A_{\text {proj }}}=\int d s_{x} d s_{y} \phi\left(s_{x}, s_{y}\right) \sqrt{1+s_{x}^{2}+s_{y}^{2}}
$$

For isotropic surfaces, $\phi\left(s_{x}, s_{y}\right)$ is rotationally symmetric. Then $\phi\left(s_{x}, s_{y}\right) d s_{x} d s_{y}=\pi \phi\left(s_{x}, 0\right)\left|s_{x}\right| d s_{x}$ with integration bounds on $s_{x}$ running from negative infinity to infinity. Hence:

$$
\frac{A_{\text {surf }}}{A_{\text {proj }}}=\pi \int_{-\infty}^{\infty}\left|s_{x}\right| \phi\left(s_{x}, 0\right) \sqrt{1+s_{x}^{2}} d s_{x}
$$

Note that the slope distribution from a line scan $\Phi\left(s_{x}\right)$ is not $\phi\left(s_{x}, 0\right)$. Since we do not know the value of the slope $s_{y}$, a line scan measures the average:

$$
\Phi\left(s_{x}\right)=\int \phi\left(s_{x}, s_{y}\right) d s_{y}
$$

i.e., the marginal distribution of slopes. By assuming that the distribution of slopes in $\mathrm{x}-$ and $\mathrm{y}-$ direction is independent (which is true for a Gaussian distribution of slopes), we can express $\phi\left(s_{x}, s_{y}\right)=\phi_{x}\left(s_{x}\right) \phi_{y}\left(s_{y}\right)$. For a Gaussian distribution of slopes we obtain:

$$
\Phi\left(s_{x}\right)=\phi_{x}\left(s_{x}\right)=\sqrt{\pi} h_{\mathrm{rms}}^{\prime} \phi\left(s_{x}, 0\right)
$$

or:

$$
\phi\left(s_{x}, 0\right)=\frac{\Phi\left(s_{x}\right)}{\sqrt{\pi} h_{\mathrm{rms}}^{\prime}}
$$

Hence, using $\int f\left(\frac{\partial h}{\partial x}\right) d x=L_{x} \int \Phi\left(s_{x}\right) f\left(s_{x}\right) d s_{x}$ we obtain:

$$
\frac{A_{\text {surf }}}{A_{\text {proj }}}=\frac{\sqrt{\pi}}{h_{\mathrm{rms}}^{\prime}} \int_{-\infty}^{\infty}\left|s_{x}\right| \Phi\left(s_{x}\right) \sqrt{1+s_{x}^{2}} d s_{x}=\frac{\sqrt{\pi} L_{y}}{h_{\mathrm{rms}}^{\prime} A_{\text {proj }}} \int\left|\frac{\partial h}{\partial x}\right| \sqrt{1+\left(\frac{\partial h}{\partial x}\right)^{2}} d x
$$

The right-hand side of Eq. S10 can be directly evaluated on a line scan, which can be discretized as follows: 


$$
\frac{A}{A_{0}}=\frac{\sqrt{\pi}}{h_{\mathrm{rms}}^{\prime} L_{x}} \sum_{i} x_{i}\left|s_{i}\right| \sqrt{1+s_{i}^{2}}=\sqrt{\frac{\pi}{2 L_{x}}} \frac{\sum_{i} x_{i}\left|s_{i}\right| \sqrt{1+s_{i}^{2}}}{\sqrt{\sum_{i} x_{i} s_{i}^{2}}}
$$

Figure S-1 compares the surface area computed using Eqs. S1, S2, and S10 for a synthetic selfaffine surface created using a Fourier filtering algorithm. ${ }^{4,5}$ As shown, Eq. S2 is appropriate for $h_{\mathrm{rms}}^{\prime} \lesssim 0.2$ and Eq. S10 for $h_{\mathrm{rms}}^{\prime} \gtrsim 0.2$.

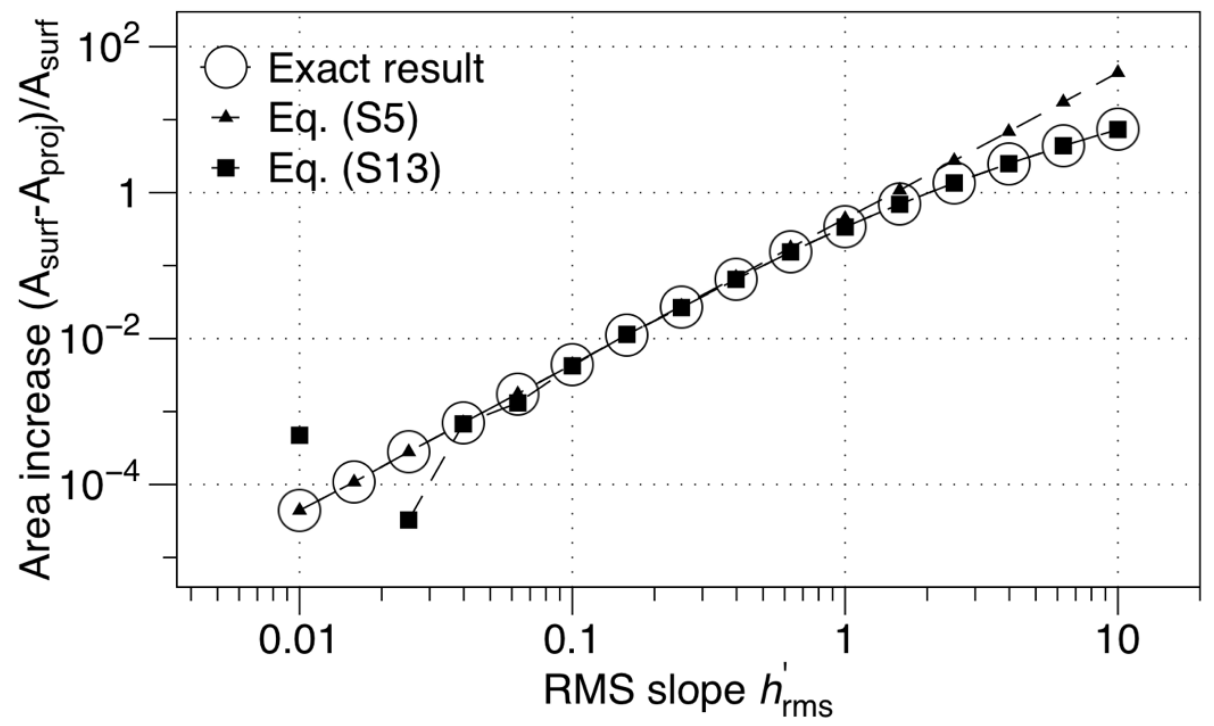

Figure S-1: Numerical comparison of equations for fractional increase in surface area. Computation of the surface area $A_{\text {proj }}$ from line scans on an ideal synthetic self-affine surface created using a Fourier filtering algorithm. The small-slope approximation (Eq. S2) gives a good prediction of the true value (Eq. S1) for $h_{\mathrm{rms}}^{\prime} \lesssim 0.2$. The large-slope approximation (Eq. S10) predicts the correct area for $h_{\mathrm{rms}}^{\prime} \gtrsim 0.2$.

\section{S-2. Showing all individually measured PSDs}

In Fig. 4 of the main text, all PSDs from the same instrument are colored in the same color and much of the data is overlapping. Therefore, it is not apparent how many data sets are represented, 
and how few datasets (3) are artifacted. The figure below shows the same data, but with all datasets colored differently.

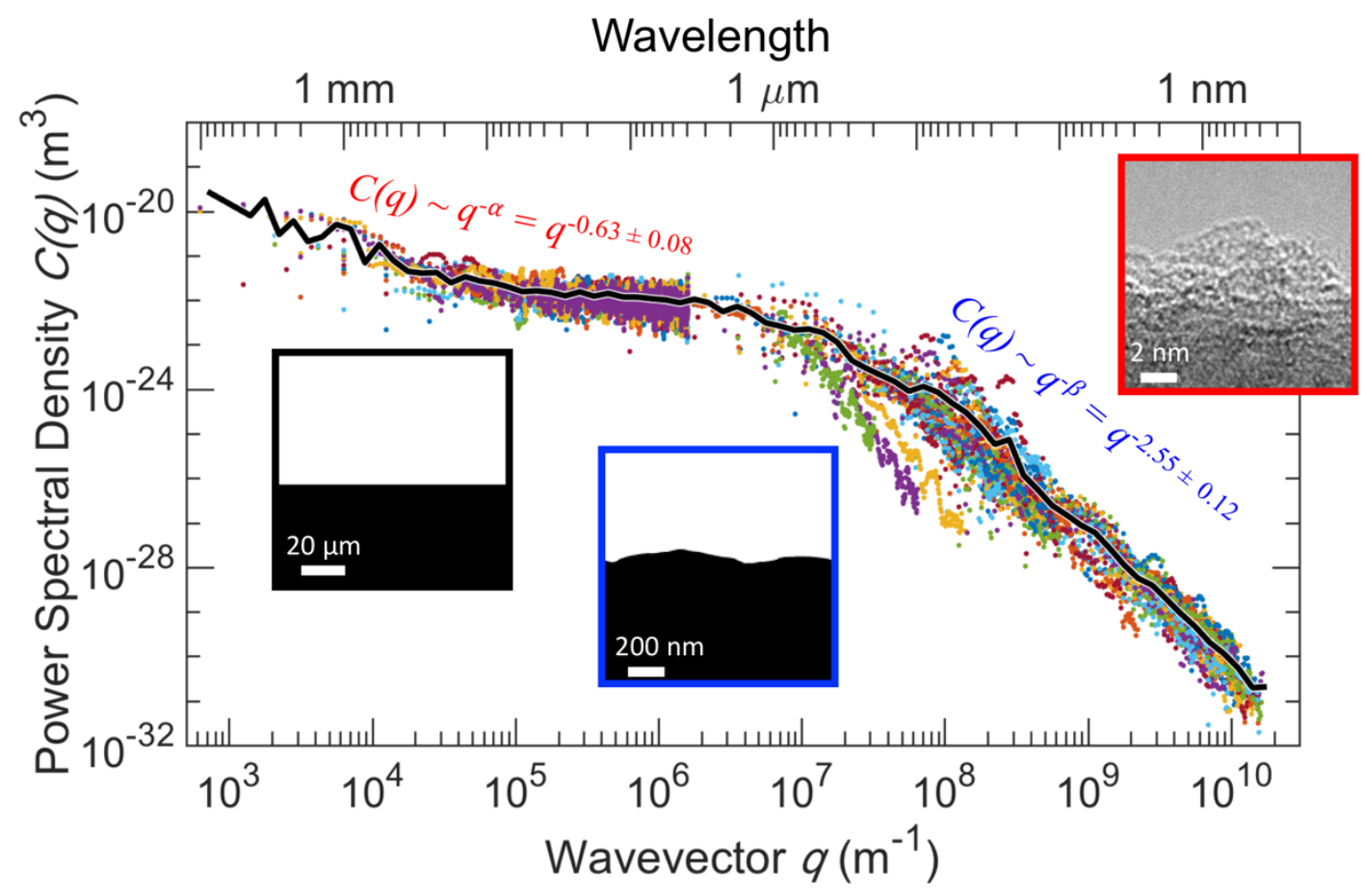

Figure S-2: Power spectral densities from individual measurements (same as Fig. 4 of main text) are shown with varying colors for clarity.

\section{S-3. A process for approximating full, multi-scale RMS parameters using only AFM data.}

In the main text, we have discussed using only AFM data to estimate RMS slope and curvature. First, a direct application of Eq. 1 can be applied to the average of all AFM measurements (red curve in Fig. S-3a). This approach is very sensitive to tip artifacts (which reduce the magnitude of the PSD) and noise artifacts (which increase the magnitude of the PSD) and leads to inaccurate values with large uncertainties. By contrast, in Fig. S-3b, the reliability analysis (Methods) is applied to the individual measurements and only the reliable data is averaged (red solid curve). Then, the latter portion of the average curve is fit to a power-law curve (straight line on a log-log plot). Using the best-fit slope and intercept of the self-affine region (subjectively 
estimated as ranging from $\mathrm{q}=1.12 \times 10^{7}$ to $1.41 \times 10^{8}$ ), the curve is extrapolated (red dashed curve) up to the largest $q$ measured in the TEM. This combined red curve was taken as an estimate of the true PSD and integrated using Eq. 1 to estimate the RMS slope and curvature. Uncertainty in the best-fit values of slope and intercept were used to compute upper and lower bounds on the extrapolated PSD, which were integrated to compute upper and lower bounds on the RMS slope and RMS curvature.
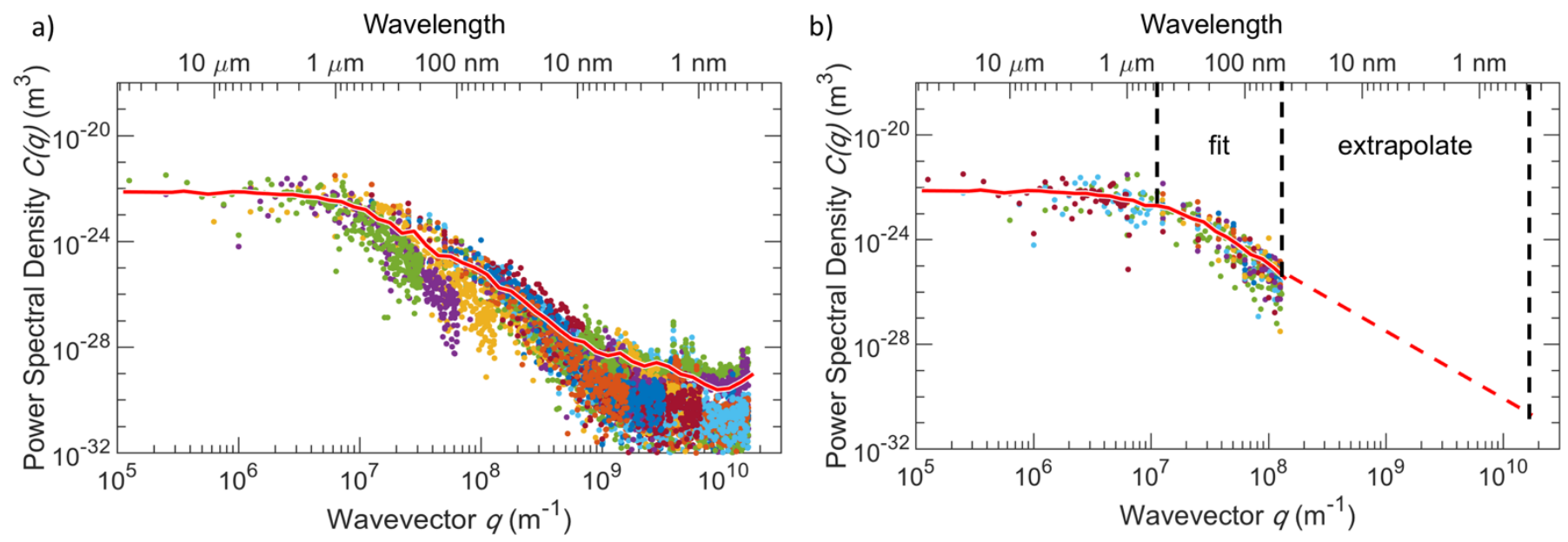

Figure S-3: The AFM data can be used in one of two ways to compute roughness parameters. In (a), all individual measurements (multi-color points) are averaged to create a single PSD (red curve), which can be integrated using Eq. 1. In (b), the average PSD (red solid line) is computed using only the non-artifacted portions of the measurements (points). Then the straight-line higher-q region is fit to a power-law function and extrapolated (red dashed line) up to the maximum q-value that was measured using TEM. Finally, this combined (solid and dashed) curve can be integrated using Eq. 1.

\section{References}

(1) Zeng, H.; Konicek, A. R.; Moldovan, N.; Mangolini, F.; Jacobs, T. D. B.; Wylie, I.; Arumugam, P. U.; Siddiqui, S.; Carpick, R. W.; Carlisle, J. A. Boron-Doped Ultrananocrystalline Diamond Synthesized with an H-rich/Ar-Lean Gas System. Carbon N. Y. 2015, 84 (1), 103-117.

(2) Jacobs, T. D. B.; Wabiszewski, G. E.; Goodman, A. J.; Carpick, R. W. Characterizing Nanoscale Scanning Probes Using Electron Microscopy: A Novel Fixture and a Practical 
Guide. Rev. Sci. Instrum. 2016, 87 (1), 13703-13711.

(3) Vahdat, V.; Carpick, R. W. Practical Method to Limit Tip-Sample Contact Stress and Prevent Wear in Amplitude Modulation Atomic Force Microscopy. ACS Nano 2013, 7 (11), 9836-9850.

(4) Jacobs, T. D. B.; Junge, T.; Pastewka, L. Quantitative Characterization of Surface Topography Using Spectral Analysis. Surf. Topogr. Metrol. Prop. 2017, 5 (1), 13001.

(5) Ramisetti, S. B.; Anciaux, G.; Molinari, J.-F. F.; Campañá, C.; Anciaux, G.; Molinari, J.F. F.; Müser, M. H.; Robbins, M. O. The Autocorrelation Function for Island Areas on Self-Affine Surfaces. 2011, 23 (21), 215004. 